\title{
Glycoprotein is enough for sindbis virus-derived DNA vector to express heterogenous genes
}

\author{
Wuyang Zhu, Jiangjiao Li, Li Tang, Huanqin Wang, Jia Li, Juanjuan Fu and Guodong Liang*
}

\begin{abstract}
To investigate the necessity and potential application of structural genes for expressing heterogenous genes from Sindbis virus-derived vector, the DNA-based expression vector pVaXJ was constructed by placing the recombinant genome of sindbis-like virus XJ-160 under the control of the human cytomegalovirus (CMV) promoter of the plasmid pVAX1, in which viral structural genes were replaced by a polylinker cassette to allow for insertion of heterologous genes. The defect helper plasmids $\mathrm{pVaE}$ or $\mathrm{pVaC}$ were developed by cloning the gene of glycoprotein E3E26KE1 or capsid protein of XJ-160 virus into PVAX1, respectively. The report gene cassette pVaXJEGFP or pV-Gluc expressing enhanced green fluorescence protein (EGFP) or Gaussia luciferase (G.luc) were constructed by cloning EGFP or G.luc gene into pVaXJ. EGFP or G.luc was expressed in the BHK-21 cells cotransfected with report gene cassettes and $\mathrm{pVaE}$ at levels that were comparable to those produced by report gene cassettes, $\mathrm{pVaC}$ and $\mathrm{pVaE}$ and were much higher than the levels produced by report gene cassette and $\mathrm{pVaC}$, suggesting that glycoprotein is enough for Sindbis virus-derived DNA vector to express heterogenous genes in host cells. The method of gene expression from Sindbis virus-based DNA vector only co-transfected with envelop $E$ gene increase the conveniency and the utility of alphavirus-based vector systems in general.
\end{abstract}

\section{Findings}

The concept that alphaviruses can be developed as expression vectors was first established by Xiong et al. [1]. Since then, Sindbis virus (SINV), a member of alphavirus, has been developed as vectors for the expression of heterologous gene [2-4], gene therapy and vaccine application [5-8]. Sindbis virus genome is a single strand of positive-sense RNA of approximately $12 \mathrm{~kb}$ which is capped at the $5^{\prime}$ terminus and polyadenylated at the 3' terminus [9]. The 5' two-thirds of this RNA encode the nonstructural proteins (nsP1 through 4). The 3' one-third is initially translated as a polyprotein (NH2-C-E3-E2-6K-E1-COOH) that is processed co- and posttranslationally to produce the structural proteins (SPs) (capsid, El and E2). In infected cells, the virion structural proteins are translated from a subgenomic mRNA (26S RNA) and produced by transcription of genome-length complementary (minus) strand from a highly active subgenomic promoter. Since the nonstructural protein genes and the structural protein genes are

\footnotetext{
* Correspondence: gdliang@hotmail.com

State Key Laboratory for Infectious Disease Prevention and Control, National Institute for Viral Disease Control and Prevention, Chinese Center for Viral Disease Control and Prevention, Beijing 100052, China
}

expressed from two different mRNAs, they may be expressed independently of one another [10]. Thus, the high levels of expression of heterologous products are achieved when the viral structural genes are replaced by the heterologous coding sequences. Such recombinant vectors are self-replicating (replicons) and can be introduced into cells as naked RNA or plasmid DNA.

As a Sindbis-like virus, XJ-160 virus (GenBank No. AF103728) was isolated from a pooled sample of Anopheles mosquitoes collected in Xinjiang, China, in 1990 [11]. Recombinant plasmid pBR-XJ160 is an infectious full-length cDNA clone of XJ-160 virus, from which rescued virus BR-XJ160 can be obtained by transcription in vitro and transfection. The BR-XJ160 virus raised in BHK21 cells was indistinguishable from the XJ-160 virus in its biological properties, including its plaque morphology, growth kinetics and suckling mouse neurovirulence [12]. On basis of pBR-XJ160, the effects of the substitutions of 169 Lys and 173Thr in nonstructural protein 1 (nsP1) as well as nsP2-726 Pro on the infectivity and pathogenesis of Sindbis virus have been investigated [13,14]. We have also confirmed the essential role of E2 glycoprotein, especially the domain of 145-150 aa, in Sindbis virus infection through the interaction with cellular heparan sulfate 
$[15,16]$. In addition, we have developed XJ-160 virus-based RNA vector system, including replicon vector pBRepXJ, a defective helper $(\mathrm{DH})$ plasmid $(\mathrm{pBR}-\mathrm{H})$ and the packaging cell lines (PCLs) $[17,18]$.

The conventional approaches producing infectious Sindbis virus RNA and its derived complementary vectors were restricted primarily to in vitro transcription of cDNA clones from a bacteriophage RNA polymerase promoter, followed by transfection into permissive cells. Compared with this method, alphavirus replication can also be initiated by transfection of plasmid DNA $[4,19]$. In this case, full-length 5'-capped RNAs are transcribed in the nucleus using a polymerase II promoter and transported to the cytoplasm, the site of primary translation and RNA amplification. Therefore, there is no need for in vitro transcription and mRNA capping as required for the transfection of previously described Sindbis virus-derived RNA vectors. For the construction of XJ160 virus-based DNA vector, the recombinant genome of XJ-160 virus was placed under the control of the human cytomegalovirus (CMV) promoter of the plasmid pVAX1 (Invitrogen, USA), in which viral structural genes were replaced by the sequence of multiple cloning site (MCS) to allow for insertion of heterologous genes (Figure 1). The virus non-structural gene sequence (17562nt), divided into three fragments XJ1 (1-2527nt),

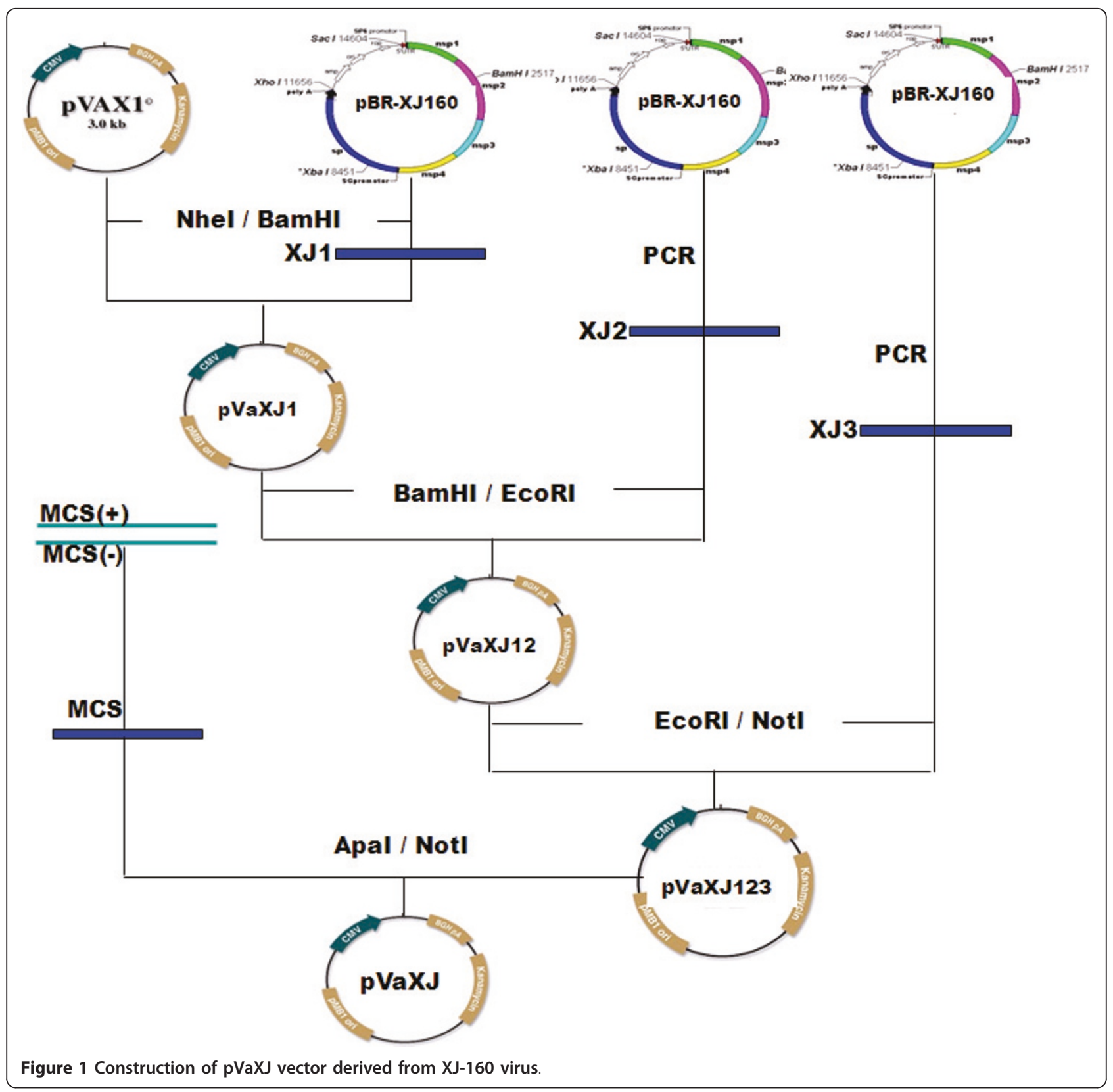


XJ2 (2527-5161nt) and XJ3 (5161-7562nt), was inserted into pVAX1 between unique NheI and NotI sites. The sequence of MCS under the control of subgenomic promoter contains several unique enzyme recognition sites, including NotI, PvuI, FseI, PacI and AscI. And the DNA vector derived from XJ-160 virus in this study was designated as pVaXJ. In addition, two DH plasmids were developed by cloning the gene of glycoprotein E3E26KE1 (8355-11297nt) or capsid protein (7563$8354 \mathrm{nt}$ ) of XJ-160 virus into pVax1, respectively.

To perform the qualitative and quantitative identification of pVaXJ, the report gene cassettes expressing either green fluorescence protein (EGFP) or Gaussia luciferase (G.luc) were constructed. In brief, the EGFP expression cassette pVaXJ-EGFP was constructed by inserting EGFP gene from the vector pEGFP-N1 (Invitrogen, USA) into the vector $\mathrm{pVaXJ}$ by digestion with NotI and AscI. Similarly, G.luc gene was amplified from pCMV-GLUC-1 vector (NEB, USA), and was inserted into $\mathrm{pVaXJ}$ with FseI and AscI.The G.luc expression cassette was designated as $\mathrm{pV}$-Gluc. To investigate the functional activity of $\mathrm{pVaXJ}$, and to determine whether both $\mathrm{DH}$ vectors are necessary to express report genes, BHK-21 cells were co-transfected with pVaXJ-EGFP $+\mathrm{pVaE}+\mathrm{pVaC}, \mathrm{pVaXJ}-\mathrm{EGFP}+\mathrm{pVaE}$ or pVaXJ-EGFP $+\mathrm{pVaC}$, respectively. And the expressions of EGFP were observed through scanned by an inverted fluorescence microscope $(400 \times)$. As can be seen in Figure 2, the green fluorescence was observed from all of three groups of BHK-21 cells at 24 hours post-cotransfection. In contrast, the control was negative. These results clearly demonstrated that XJ-160 virus-based DNA vector (pVaXJ) was able to efficiently express report genes by co-transfection with $\mathrm{pVaE}, \mathrm{pVaC}$ or both of them,
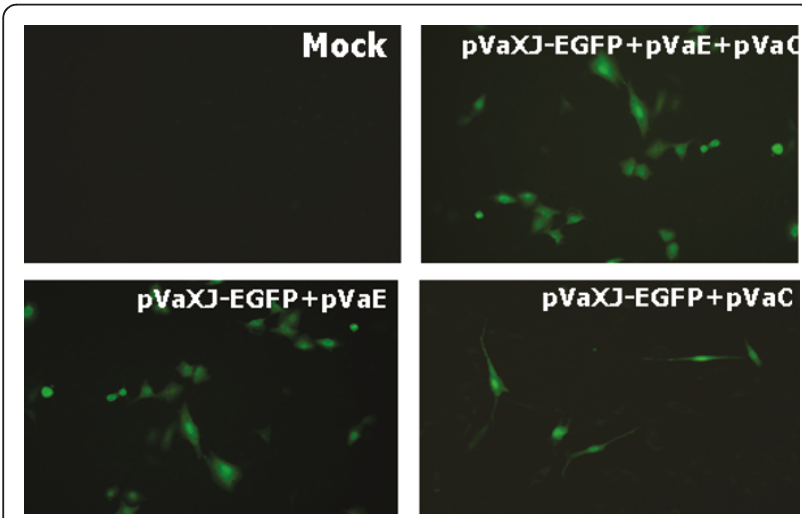

Figure 2 Expression of EGFP on BHK-21 cells. BHK-21 cells were cotransfected with pVaXJ-EGFP+pVaE+pVaC, pVaXJ-EGFP+pVaE, pVaXJ-EGFP+pVaC, respectively, and the expressions of EGFP were observed through scanned by an inverted fluorescence microscope (400x) at 24 hours postinfection. The BHK-21 cells without any transfection (Mock) were used as control. respectively. Importantly, the level of specific fluorescence from the BHK-21 cells co-transfected with pVaXJ-EGFP+pVaE was comparable to that produced by $\mathrm{pVaXJ}-\mathrm{EGFP}+\mathrm{pVaC}+\mathrm{pVaE}$, and obviously higher than that from the BHK-21 cells co-transfected with $\mathrm{pVaXJ-EGFP} \mathrm{and} \mathrm{pVaC}$, suggesting that glycoprotein plays a more important role than capsid protein in expressing heterogenous gene from $\mathrm{pVaXJ}$ vector.

For further comparing the necessity and potential application of structural genes, expression of G.luc gene was detected after BHK-21 cells were co-transfected with pV-Gluc, pV-Gluc $+p V a E+p V a C, p V-G l u c+p V a E$ or $\mathrm{pV}-\mathrm{Gluc}+\mathrm{pVaC}$, respectively. Gluc assay was performed by Gaussia Luciferase Assay System (Promega) following the manufacturer's instructions. As shown in Figure 3, G.luc gene was efficiently expressed in all groups of BHK-21 cells except that transfected with pV-Gluc plasmid, indicating that either glycoprotein or capsid protein is important for $\mathrm{pVaXJ}$ to express report genes. Interestingly, the activity of G.luc observed from the BHK-21 transfected with $\mathrm{pV}-\mathrm{Gluc}+\mathrm{pVaE}$ was much higher than that from the BHK-21 cells co-transfected with pV-Gluc $+\mathrm{pVaE}+\mathrm{pVaC}$, whose expression efficiency was similar to that of the BHK-21 cells transfected with pV-Gluc $+\mathrm{pVaC}$. As mentioned earlier, the alphavirus particles are surrounded be a lipid bilayer containing envelop glycoprotein heterodimer. And the capsid subunits complex with the genome RNA to form a nucleocapsid acquiring the lipid envelop with embedded viral glycoproteins. In addition, the capsid protein of alphaviruses may be important factor in the inhibitionof host cell protein synthesis. Therefore, compared with capsid, glycoprotein plays a more important role in packaging virus particles or virus like particles, and is enough for

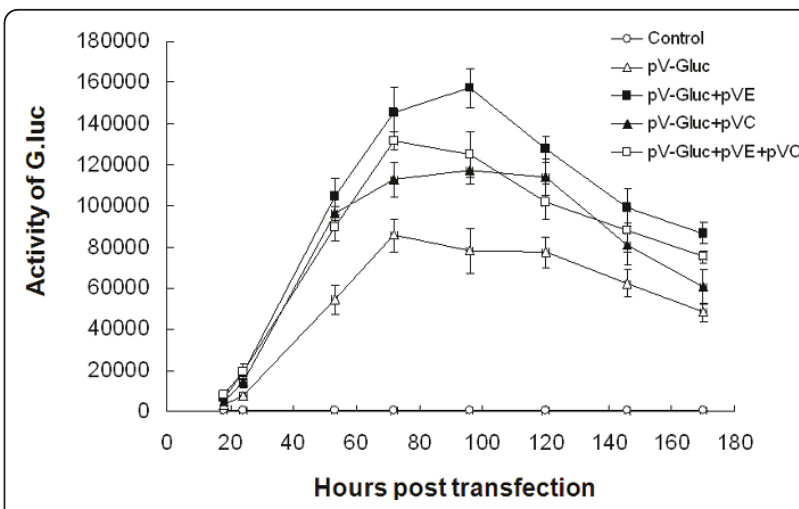

Figure 3 Expression of G.luc on BHK-21cells. BHK-21 cells were cotransfected with pV-Gluc, pV-Gluc +pVaE, pV-Gluc +pVaC or pVGluc $+p V a E+p V a C$, respectively. Gluc assay was performed by Gaussia Luciferase Assay System (Promega) at the indicated time postinfection. 
Sindbis virus-derived DNA vector to express heterogenous genes in host cells.

DNA-based vector derived from alphavirus can be initiated by transfection of plasmid DNA [4,19]. In this strategy, full-length 5'-capped RNAs are transcribed in the nucleus using a polymerase II promoter, and transported to the cytoplasm. Then autocatalytic amplification of the vector, proceeds according to the Sindbis virus replication cycle and results in expression of the heterologous gene. Thus, as a double-stranded DNA, DNA-based vector can be directly transfected into the host cells dispensing with the process of in vitro transcription and mRNA capping, which is required for the transfection of previously described Sindbis virusderived RNA vectors. In this study, we have constructed DNA-based vector pVaXJ from XJ-160 virus, which could efficiently express reporter genes in the transfected cells. The XJ-160 virus-derived DNA vectors described here increase the utility of alphavirus-based vector systems in general and also provide a vector with broad potential applications for genetic immunization.

The SINV-derived replicons lacking viral structural genes are suicide vectors, incapable of packaging into infectious particles and causing productive infection. To obtain viable particles, vector replicon can be packaged into infectious particles by cotransfection of cultured cells with a defective helper vector, which provides the virion structural proteins in trans [20]. Two principal strategies are being performed for construction of DH vector: (i) engineering recombinant plasmid that expresses viral subgenomic RNAs (NH2-C-E3-E2-6K$\mathrm{E} 1-\mathrm{COOH}$ ) and (ii) developing two $\mathrm{DH}$ vectors, one that expresses the capsid protein and a second that expresses the viral glycoproteins. High levels of expression of heterologous genes were achieved by using both of two methods. However, it is not clear which of the two SPs or both is necessary for SINV-derived vector to express heterologous coding sequences. In this investigation, based on the XJ-160 virus-derived DNA vector, our findings clearly prove that glycoprotein is enough for Sindbis virus-derived DNA vector to express heterogenous genes in host cells. The method of gene expression from Sindbis virus-based DNA vector only co-transfected with envelop $\mathrm{E}$ gene increases the conveniency and the utility of alphavirus-based vector systems in general.

\section{Acknowledgements}

This work was supported by the grants from the National Natural Science Foundation of China (No. 30970160), the Development Grant of State Key Laboratory for Infectious Disease Prevention and Control (2011SKLID205), the Major Science and Technology Project for Infectious Disease (No.

2008ZX10004-001; 2009ZX10004-705)

\section{Authors' contributions}

WZ carried out the molecular genetic studies, participated in the sequence alignment and drafted the manuscript. LT and $J \mathrm{~L}$ carried out the

immunoassays. HW, JL and JF participated in the sequence alignment and performed the statistical analysis. GL conceived of the study, and participated in its design and coordination. All authors read and approved the final manuscript.

\section{Competing interests}

The authors declare that they have no competing interests.

Received: 14 May 2011 Accepted: 10 July 2011 Published: 10 July 2011

\section{References}

1. Xiong C, Levis R, Shen P, Schlesinger S, Rice CM, Huang HV: Sindbis virus: an efficient, broad host range vector for gene expression in animal cells. Science 1989, 243:1188-1191.

2. Bredenbeek P, Rice CM: Animal RNA virus expression systems. Semin Virol 1992, 3:297-310.

3. Schlesinger S: Alphaviruses-vectors for the expression of heterologous genes. Trends Biotechnol 1993, 11:18-22.

4. Frolov I, Hoffman TA, Pragai BM, Dryga SA, Huang HV, Schlesinger S, Rice and CM: Alphavirus-based expression vectors: strategies and applications. Proc Natl Acad Sci USA 1996, 93:11371-11377.

5. Hariharan MJ, Driver DA, Townsend K, Brumm D, Polo JM, Belli BA, Catton DJ, Hsu D, Mittelstaedt D, McCormack JE, Karavodin L, Dubensky TW, Chang SM Jr, Banks TA: DNA immunization against herpes simplex virus: enhanced efficacy using a Sindbis virusbased vector. J Virol 1998, 72:950-958.

6. Perri S, Greer CE, Thudium K, Barbara D, Legg H, Liu H, Romero RE, Tang ZQ, Bin Q, Dubensky TW, Vajdy JM, Otten GR, Polo JM: An alphavirus replicon particle chimera derived from venezuelan equine encephalitis and sindbis viruses is a potent gene-based vaccine delivery vector. $J$ Virol 2003, 77:10394-10403

7. Pan CH, Valsamakis A, Colella T, Nair N, Adams RJ, Polack FP, Greer CE, Perri S, Polo JM, Griffin DE: Modulation of disease, T cell responses, and measles virus clearance in monkeys vaccinated with $\mathrm{H}$-encoding alphavirus replicon particles. Proc Natl Acad Sci USA 2005, 115811-11588.

8. Lin YL, Kwon T, Polo J, Zhu YF, Coates S, Crawford K, Dong C, Wininger M, Hall J, Selby M, Coit D, Medina-Selby A, McCoin C, Ng P, Drane D, Chien D, Han J, Vajdy M, Houghton M: Induction of broad CD4+ and CD8+ T-Cell responses and cross-neutralizing antibodies against hepatitis $C$ virus by vaccination with Th1-adjuvanted polypeptides followed by defective alphaviral particles expressing envelope glycoproteins gpE1 and gpE2 and nonstructural proteins 3, 4, and 5. J Virol 2008, 82:7492-7503.

9. Strauss JH, Strauss EG: Structure and replication of the Alphavirus genome. In The Togaviridae and Flaviviridae. Edited by: Schlesinger S Schlesinger MJ. Plenum Press, New York; 1986:35-82.

10. Strauss JH, Strauss EG: The alphaviruses: gene expression, replication, and evolution. Microbiol Rev 1994, 58:491-562.

11. Liang GD, Li L, Zhou GL, Fu SH, Li QP, Li FS, He HH, Jin Q, He Y, Chen BQ Hou YD: Isolation and complete nucleotide sequence of a Chinese Sindbis-like virus. J Gen Virol 2000, 81:1347-1351.

12. Yang YL, Liang GD, Fu SH, Tang Q, Wang LH: Construction and infection analysis of the full-length cDNA clone of $\mathrm{XJ}-160$ virus, the first Sindbis virus isolated in China. Virol Sin 2005, 20:173-180.

13. Zhu WY, Yang YL, Fu SH, Wang LH, Zhai YG, Tang Q, Liang GD: Substitutions of 169Lys and 173Thr in nonstructural protein 1 influence the infectivity and pathogenicity of XJ-160 virus. Arch Virol 2009, 154:245-253.

14. Zhu WY, Fu SH, Wang JL, He Y, Tang Q, Liang GD: Effects of the nsP2-726 Pro mutation on infectivity and pathogenesis of Sindbis virus derived from a full-length infectious cDNA clone. Virus Res 2009, 14:204-207.

15. Zhu W, Wang L, Yang Y, Jia J, Fu S, He Y, Li JP, Liang G: Interaction of E2 glycoprotein with heparan sulfate is crucial for cellular infection of sindbis virus. PLOS ONE 2010, 5(3):e9656.

16. Zhu WY, Fu SH, Li JP, He Y, Liang GD: Amino acid substitutions in the E2 glycoprotein of Sindbis-like virus XJ-160 confer the ability to undergo heparan sulfate-dependent infection of mouse embryonic fibroblasts. Virol J 2010, 7:225 
17. Yang YL, Liang GD, Fu SH, Liang GD: The construction of replicon of XJ160 virus. Virol $\operatorname{Sin} 2003,18: 221-226$.

18. Zhu WY, Liang GD: Selection and characterization of packaging cell lines for XJ-160 Virus. Intervirology 2009, 52:100-109.

19. Dubensky TW, Driver DA Jr, Polo JM, Belli BA, Latham EM, Ibanez CE, Chada S, Brumm D, Banks TA, Mento SJ, Jolly DJ, Chang SMW: Sindbis virus DNA-based expression vectors: utility for in vitro and in vivo gene transfer. J Virol 1996, 70:508-519.

20. Bredenbeek PJ, Frolov I, Rice CM, Schlesinger S: Sindbis virus expression vectors: packaging of RNA replicons by using defective helper RNAs. $J$ Virol 1993, 67:6439-6446.

doi:10.1186/1743-422X-8-344

Cite this article as: Zhu et al.: Glycoprotein is enough for sindbis virusderived DNA vector to express heterogenous genes. Virology Journal $20118: 344$

\section{Submit your next manuscript to BioMed Central} and take full advantage of:

- Convenient online submission

- Thorough peer review

- No space constraints or color figure charges

- Immediate publication on acceptance

- Inclusion in PubMed, CAS, Scopus and Google Scholar

- Research which is freely available for redistribution

Submit your manuscript at www.biomedcentral.com/submit 